\title{
Efecto de la interacción luz-agua sobre la fotosíntesis de la Vanilla planifolia (Orchidaceae)
}

\author{
E. Alberto Gantiva R. ${ }^{1 *}$, M. Claudia Díez G. ${ }^{2}$ \& Flavio H. Moreno H. ${ }^{3}$ \\ 1. Consultor independiente proyectos productivos y cultivador de vainilla, Colombia; albertogantiva@hotmail.com \\ 2. Departamento de Ciencias Forestales, Universidad Nacional de Colombia, sede Medellín, Colombia; \\ mcdiez@unal.edu.co \\ 3. Departamento de Ciencias Forestales, Universidad Nacional de Colombia, sede Medellín, Colombia; \\ fhmoreno@unal.edu.co \\ * Correspondencia
}

Recibido 11-IV-2020. Corregido 26-VII-2020. Aceptado 31-VIII-2020.

\begin{abstract}
Light-water interaction effect on photosynthesis of the Vanilla planifolia (Orchidaceae). Introduction: Vanilla planifolia Andrews is a CAM plant of economic importance in the global market of flavorings and essences. Being a hemiepiphyte which initially grows in the shady understory and later climbs to the canopy, it acclimates to different conditions of radiation and humidity. The possibility of extending this crop to dry areas where the incidence of diseases is lower will depend on the response to prolonged periods of drought. Objective: To evaluate the effects of the interaction of radiation and humidity on the parameters of photosynthesis and operation of CAM of vanilla plants, and to determine if the radiation environment could help to moderate the negative effects of drought. Methods: We evaluated well-watered vanilla plants grown for 18 months in relative illumination (RI) of $8,17,31$ and $67 \%$, which underwent dehydration for 94 days until reaching critical water content (relative water content, RWC $<50 \%$ ) and then rehydration for 22 days; variables evaluated were: $\mathrm{CO}_{2}$ assimilation, titratable acidity, and quantum efficiency of Photosystem II (Fv / Fm). Results: Under intermediate conditions of radiation (17\% and $31 \% \mathrm{RI}), \mathrm{CO}_{2}$ assimilation and nocturnal accumulation of organic acids were less affected by water deficit and also showed the best recovery after rehydration. The RWC was most affected by the water available in plant tissue, while Fv / Fm was affected by radiation. The combined effects of water stress and high radiation caused irreversible damage to photosynthesis for the $67 \%$ RI treatment. Conclusions: In vanilla plants, the negative impact of drought on photosynthetic parameters was greater in high radiation; however, in low radiation conditions the susceptibility of plants to drought also increased, as compared to intermediate radiation environments, which were under a mean photon flux density of $340 \mu \mathrm{mol} \mathrm{m}^{-2} \mathrm{~s}^{-1}(17 \%$ IR) and $620 \mu \mathrm{mol} \mathrm{m}^{-2} \mathrm{~s}^{-1}(31 \% \mathrm{IR})$. These results suggest the potential for growing vanilla in areas with seasonal drought under low-tech production systems, maintaining these radiation conditions.
\end{abstract}

Key words: titratable acidity; CAM; relative water content; water deficit; chlorophyll fluorescence; photosynthesis; Orchidaceae.

Gantiva R., E.A., Díez G., M.C., \& Moreno H., F.H. (2020). Efecto de la interacción luz-agua sobre la fotosíntesis de la Vanilla planifolia (Orchidaceae). Revista de Biología Tropical, 68(4), 1250-1261.

La vainilla (Vanilla planifolia Andrews) es una orquídea hemiepífita de los bosques neotropicales que se desarrolla en diferentes niveles de radiación a lo largo de su ciclo de vida. Inicialmente crece en el sotobosque sombreado y luego, apoyándose en los árboles, llega al dosel donde florece y fructifica (Fouché \& Jouve, 1999). Además, está expuesta continuamente a variaciones espaciales y temporales del ambiente, por lo cual se espera que tenga 
una alta capacidad de aclimatación a diferentes ambientes de radiación y disponibilidad de agua, al igual que otras epífitas de los bosques tropicales (Zotz \& Hietz, 2001).

Es frecuente que las epífitas que crecen en el dosel de los bosques tropicales experimenten déficit hídrico durante algunos períodos del año, lo cual explicaría que cerca del $19 \%$ de estas plantas expresan un funcionamiento y fotosíntesis tipo CAM (Zotz, 2004), entre ellas, las orquídeas del género Vanilla (Buss, 2020). Este metabolismo, que se considera una adaptación eco fisiológica a la escasez de agua (Silvera \& Lasso, 2016) se caracteriza por tomar $\mathrm{CO}_{2}$ de la atmósfera predominantemente durante la noche, con lo cual logra la máxima ganancia de carbono combinada con mínima pérdida de agua (Winter, 2019). Las plantas CAM tienen alta plasticidad, ya que pueden cambiar el patrón del ciclo de la fotosíntesis en respuesta a claves ambientales, como la sequía (Dodd, Borland, Haslam, Griffiths, \& Maxwell, 2002). Así, en las plantas que experimentan estrés hídrico, la toma de $\mathrm{CO}_{2}$ diurna se reduce y después de una sequía prolongada, llega a ser exclusivamente nocturnas ( $\mathrm{Fu} \&$ Hew, 1982). Por otra parte, la radiación en la que crecen las plantas también afecta la fotosíntesis, pues la magnitud de la acumulación de ácidos orgánicos durante la noche está relacionada con la intensidad de la radiación en el día previo (Borland, Maxwell, \& Griffiths, 2000).

La vainilla se cultiva en varios países tropicales debido a su importancia y alto valor económico en el mercado internacional de saborizantes y aromatizantes (Bory, Grisoni, Duval, \& Besse, 2008). El éxito de este cultivo depende, principalmente, del manejo apropiado de la radiación y la disponibilidad de agua, por su gran influencia sobre la fotosíntesis, la sobrevivencia, el crecimiento, así como de la habilidad de aclimatación a diferentes zonas agroecológicas (Puthur, 2005).

En estudios anteriores se evaluaron las respuestas de las plantas de vainilla a cambios en una sola de estas variables (radiación o disponibilidad de agua), mientras la otra se mantiene constante (Ramírez, Rapidel, \& Mattey, 1999;
Puthur, 2005; Díez, Moreno, \& Gantiva, 2017). Pero dado que la respuesta combinada a dos o más factores no se puede predecir a partir de las respuestas individuales, estos estudios no son suficientes para comprender los patrones naturales que se caracterizan por variaciones simultáneas en diferentes variables ambientales (Tay, He, \& Yam, 2019). El estudio de esta interacción en vainilla tiene gran importancia para orientar su cultivo, sobre todo para definir las estrategias de manejo cuando se planta en zonas de bosque seco tropical, donde ocurre una estación seca prolongada, lo cual a su vez podría disminuir la incidencia de patógenos, que es el principal factor limitante en la producción de vainilla en todo el mundo (Kadir, Naher, \& Sidek, 2019).

Se han planteado tres predicciones con respecto a la interacción de la radiación y la disponibilidad de agua sobre el crecimiento y desempeño de las plantas (Cavatte et al., 2012). La primera predicción plantea que, en ambientes con baja radiación, la sequía tendrá menores efectos negativos, porque en la sombra, las temperaturas del aire y de la hoja y el déficit de presión de vapor son más bajos, favoreciendo la hidratación de las plantas (de la Rosa-Manzano, Andrade, Zotz, \& Reyes-García, 2017). La segunda predicción, por el contrario, indica que, en ambientes con baja radiación, la sequía tendrá mayores efectos negativos, debido a la competencia por la asignación de biomasa, bien sea a las hojas o a las raíces, en respuesta a la necesidad de interceptar radiación $v s$. capturar agua del suelo (Haslam, Borland, Maxwell, \& Griffiths, 2003). La tercera predicción considera que el efecto de la cantidad de radiación y la sequía son independientes, ya que la sequía reduce el desempeño de las plantas en una proporción dada, en cualquier cantidad de radiación (Graham \& Andrade, 2004).

El objetivo de este trabajo fue evaluar los efectos de la interacción de la radiación y la disponibilidad de agua sobre los parámetros de la fotosíntesis y funcionamiento del CAM en plantas de vainilla y determinar si las condiciones de radiación en las que crece la planta pueden ayudar a moderar los efectos negativos 
de la sequía. Puesto que la vainilla es una planta tolerante a la sombra, se espera que el impacto negativo de la sequía sobre las plantas sea muy fuerte a alta radiación. Asimismo, en ambientes muy sombreados, la baja energía disponible para la fotosíntesis, unida a la competencia por recursos entre las porciones aérea y subterránea, aumentará la susceptibilidad de las plantas a la sequía, en comparación con el ambiente de sombra intermedia. Es decir, la predicción de este trabajo es que los efectos de la sequía sobre la fotosíntesis y funcionamiento del CAM de la vainilla serán menores en condiciones intermedias de radiación.

\section{MATERIALES Y MÉTODOS}

Área experimental: El ensayo se estableció en terrazas aluviales de relieve plano, en el valle interandino del río Cauca (Santa $\mathrm{Fe}$ de Antioquia, Colombia, 6033'32" N \& 7704'51" W), a una altitud de $540 \mathrm{~m}$, en predios del Centro Agropecuario Cotové, propiedad de la Universidad Nacional de Colombia. La temperatura promedia anual es $27{ }^{\circ} \mathrm{C}$, precipitación promedia anual es $1058 \mathrm{~mm}$ y la humedad relativa del aire promedia es $75 \%$ (Estación Hacienda Cotové, IDEAM).

Establecimiento del ensayo: Se utilizaron esquejes sanos de Vanilla planifolia provenientes de un cultivo cercano, de $80 \pm 10 \mathrm{~cm}$ de longitud y $1.5 \pm 0.5 \mathrm{~cm}$ de diámetro. Cada esqueje se sembró en un recipiente plástico (capacidad 5 1) con un tutor de madera de $150 \mathrm{~cm}$ de altura, y un sustrato compuesto por cáscara de coco + suelo orgánico + madera fragmentada + hojarasca (proporción en volumen 4:2:1:1). Se evaluaron cuatro tratamientos de radiación para lo cual se construyeron 16 casetas (cuatro por tratamiento) de $100 \mathrm{~cm}$ de largo x $80 \mathrm{~cm}$ de ancho y $300 \mathrm{~cm}$ de altura, separadas 120 cm para evitar el sombreamiento entre ellas; las casetas se cubrieron con capas de polisombra color negro de sombra neutra (Tesicol®) hasta obtener la condición de radiación requerida y en cada una se distribuyeron seis plantas de vainilla (24 plantas por tratamiento y 96 plantas en total). La radiación dentro y fuera de las casetas se midió con sensores cuánticos (LICOR, LI-190, Lincoln, Nebraska, USA) conectados a un almacenador de datos (LI-1400). Se tomaron datos a las 9:00, 13:00 y 16:00 h, y se calculó el promedio de estas tres mediciones. De esta manera los tratamientos de radiación fueron: $1340 \mu \mathrm{mol} \mathrm{m}{ }^{-2} \mathrm{seg}^{-1}(67 \%$ de la radiación a plena exposición), $620 \mu \mathrm{mol}$ $\mathrm{m}^{-2} \mathrm{seg}^{-1}$ (31\% IR), $340 \mu \mathrm{mol} \mathrm{m} \mathrm{m}^{-2} \mathrm{seg}^{-1}(17 \%$ de IR) y $160 \mu \mathrm{mol} \mathrm{m}^{-2} \mathrm{seg}^{-1}(8 \%$ de IR), considerando que la iluminación a plena exposición fue $2000 \mu \mathrm{mol} \mathrm{m}^{-2} \mathrm{seg}^{-1}$.

Las plantas crecieron en cada uno de estos niveles de radiación durante 18 meses, con riego periódico para conservar alta hidratación de los tejidos. Después de este período de aclimatación, se empezó la suspensión del riego. Para esto, se construyó un techo de plástico transparente de $6 \mathrm{~m}$ de altura sobre las casetas, con el fin de evitar ingreso de agua por precipitación. La suspensión del riego se mantuvo hasta que las plantas de todos los tratamientos alcanzaron un valor crítico de humedad en el tejido foliar (50\% de Contenido Hídrico Relativo - CHR), que fue de 94 días para el tratamiento que más se demoró, y finalmente se restableció el riego de todas las plantas durante 22 días.

Medición del Contenido Hídrico Relativo (CHR): Los cambios en la humedad de las hojas se monitorearon cada semana, durante todo el período de suspensión y restablecimiento del riego, siguiendo el protocolo de Munns (2010). Para esto, se escogieron cuatro plantas al azar de cada tratamiento de radiación (una por caseta) y en la mañana (07:00 h) se extrajeron discos de tejido foliar en hojas jóvenes y completamente desarrolladas, ubicadas entre la segunda y séptima posición desde el ápice hacia la base de la planta. A los discos de tejido foliar se les tomó el peso fresco, se hidrataron hasta saturación sumergiéndolos en agua destilada durante $8 \mathrm{~h}$ para encontrar el peso del tejido saturado, y finalmente se secaron al horno a $70{ }^{\circ} \mathrm{C}$ durante $48 \mathrm{~h}$ para determinar el peso seco. El CHR se calculó como (peso tejido 
fresco - peso tejido seco) / (peso tejido saturado - peso tejido seco).

Evaluación de la fotosíntesis y funcionamiento del CAM: Las variables asimilación de $\mathrm{CO} 2$, acidez titulable y eficiencia fotosintética se midieron en estas fases del ensayo: i) al inicio, antes de la deshidratación (CHR > $90 \%$ ), ii) después de $48 \mathrm{~d}$ de deshidratación, iii) cuando las plantas de cada tratamiento de iluminación alcanzaron un valor crítico de contenido de humedad (50\% de CHR) y iv) al final del ensayo después de un período de rehidratación de 22 días. La medición de estos parámetros se efectuó en hojas jóvenes y completamente desarrolladas, ubicadas entre la tercera y séptima posición desde el ápice hacia la base de la planta.

Medición de la asimilación de $\mathrm{CO}_{2}$ : Se seleccionaron al azar cuatro plantas por tratamiento de radiación (una por caseta) y en cada planta se escogió una hoja con las características ya descritas. Se midió el intercambio de gases cada $2 \mathrm{~h}$ durante ciclos de $24 \mathrm{~h}$ con un analizador infrarrojo de gases Li-6400 XT (Li-COR, Lincoln, Nebraska, USA). En la calibración del equipo se fijó el flujo de aire en $500 \mu \mathrm{mol} \mathrm{s}^{-1}$ y la temperatura de bloque en 27 ${ }^{\circ} \mathrm{C}$. Con estos datos se calculó la cantidad total de $\mathrm{CO}_{2}$ absorbido por las plantas de cada tratamiento de iluminación durante todo el ciclo de medición $(24 \mathrm{~h})$, durante la noche $(18: 00 \mathrm{~h}$ a 06:00 h) y durante el día (06:00 h a 18:00 h), como la integración del área bajo la curva de asimilación en cada caso.

Medición de la acidez titulable: Para su evaluación se seleccionaron al azar cinco plantas por tratamiento de radiación. Se tomaron muestras al final del período de luz del día (en la tarde entre 17:45 y 18:30 h) y al final del período de oscuridad del día siguiente (en la mañana entre 05:30 y 06:30 h), para calcular la acumulación nocturna de ácidos como la diferencia en la acidez titulable del tejido foliar entre estas dos mediciones. En cada muestreo se extrajeron dos discos de tejido foliar (de $1.2 \mathrm{~cm}$ de diámetro cada uno) de las hojas preseleccionadas, colectados de la parte central de la lámina de la hoja. Inmediatamente después de su extracción, los discos se pesaron (peso fresco) y se almacenaron en nitrógeno líquido; en el laboratorio se guardaron en un contenedor opaco a $-80{ }^{\circ} \mathrm{C}$. Para el proceso de titulación, las muestras de tejido foliar se hirvieron en etanol al $20 \%$, y la acidez titulable se midió como la cantidad de $\mathrm{nM} \mathrm{NaOH}$ que se requiere para neutralizar el extracto hasta un $\mathrm{pH}$ de 7.0, medido con un pHmetro (Hanna 211, Woonsocket, RI, USA) (Silvera et al., 2005), y se expresó por unidad de masa foliar fresca, calculada mediante la siguiente ecuación:

$\mu e q \mathrm{H}^{+}=\frac{(\mathrm{ml} \mathrm{NaOH}(\text { para alcanzar } \mathrm{pH} 7) *(\mathrm{mM} \text { solución utilizada })}{\text { Peso fresco de la muestra }(\mathrm{g})}$

Medición de la eficiencia fotosintética: Se determinó la fluorescencia de la clorofila con un fluorómetro óptico (OS-30p, Opti Sciences, USA). Su medición se efectuó en las mismas plantas seleccionadas para la medición del intercambio de $\mathrm{CO}_{2}$. Las mediciones se hicieron en la mañana (07:00 h). Antes de cada medición, la lámina foliar se oscureció durante $20 \mathrm{~min}$, mediante la instalación de pinzas de oscurecimiento; posteriormente, para la medición, se utilizó un rayo de luz actínica de alta intensidad $\left(6000 \mu \mathrm{mol} \mathrm{m}{ }^{2} \mathrm{seg}^{-1}\right)$ durante $2 \mathrm{~s}$. El equipo calculó automáticamente la Eficiencia cuántica del fotosistema II (Fv/Fm).

\section{Diseño experimental y análisis estadísti-}

co: En los conjuntos de datos obtenidos en cada parámetro, se inspeccionaron los supuestos de normalidad y homocedasticidad mediante pruebas de bondad de ajuste (Shapiro-Wilk, Kolmogorov-Smirnov e histogramas de frecuencia). El efecto de la radiación en las diferentes fases de deshidratación y rehidratación de las plantas, sobre los parámetros contenido hídrico relativo, asimilación de $\mathrm{CO}_{2}$, acidez titulable y eficiencia cuántica, se evaluaron mediante análisis de varianza y pruebas de rangos múltiples. En todos los casos, las diferencias estadísticas entre los tratamientos se establecieron mediante 
una prueba de Duncan. Los análisis estadísticos se ejecutaron en el programa Statgraphics-XVI.

\section{RESULTADOS}

\section{Cambios en el contenido hídrico relativo} durante las fases de deshidratación y rehidratación de las plantas: Al inicio del ensayo las plantas de todos los tratamientos de iluminación relativa tenían contenidos hídricos del tejido foliar altos (por encima del $93 \%$ CHR), sin diferencias significativas entre ellos $(\mathrm{P} \leq$ 0.05, $\mathrm{N}=5$ ) (Tabla 1 y Fig. 1 ).

Cuando las plantas se dejaron de regar, el punto crítico de deshidratación (CHR $\leq 50$ $\% \pm 3$ ) lo alcanzó primero el tratamiento con mayor radiación, seguido por los tratamientos de iluminación intermedia, y posteriormente el tratamiento más oscuro (a los 48, 70 y 94 días respectivamente). Luego, las plantas se rehidrataron hasta el día 115 del inicio del ensayo, que correspondió a 22 días después de que se reinició el riego en el tratamiento de menor radiación. La tendencia general mostró un aumento rápido y significativo del $\mathrm{CHR}$ en todos los tratamientos (Tabla 1), llegando nuevamente a valores por encima del $90 \%$ del CHR, excepto el tratamiento de mayor radiación, que fue significativamente inferior a los demás tratamientos.

\section{Cambios en la asimilación de $\mathrm{CO}_{2}$} durante las fases de deshidratación y rehidratación de las plantas: $\mathrm{Al}$ inicio del ensayo, durante la fase de hidratación óptima (CHR $\geq$ $90 \%$ ), la asimilación de $\mathrm{CO}_{2}$ diurna (06:00 h a 18:00 h) fue diferente entre el tratamiento más oscuro (8\% IR) y el de mayor radiación (67\% IR); no hubo diferencias entre los demás tratamientos. Los valores de asimilación de $\mathrm{CO}_{2}$ nocturna (18:00 h a 06:00 h) de los tratamientos intermedios fueron significativamente mayores $(\mathrm{P} \leq 0.05)$ del de mayor radiación, y los tratamientos extremos no presentaron diferencias entre sí. Esta tendencia se mantuvo en el balance durante las 24 horas (Fig. 2A y Tabla 1).

Después de 48 días sin riego, la asimilación diurna de $\mathrm{CO}_{2}$ disminuyó considerablemente en todos los tratamientos, llegando a valores negativos; los tratamientos intermedios tuvieron valores significativamente superiores a los de los tratamientos de radiación muy baja o muy alta. La asimilación nocturna de $\mathrm{CO}_{2}$ para este período muestra un mayor contraste entre el tratamiento de menor y el de mayor radiación, pues el primero presentó mayor asimilación y el segundo, mayor respiración (Tabla 1).

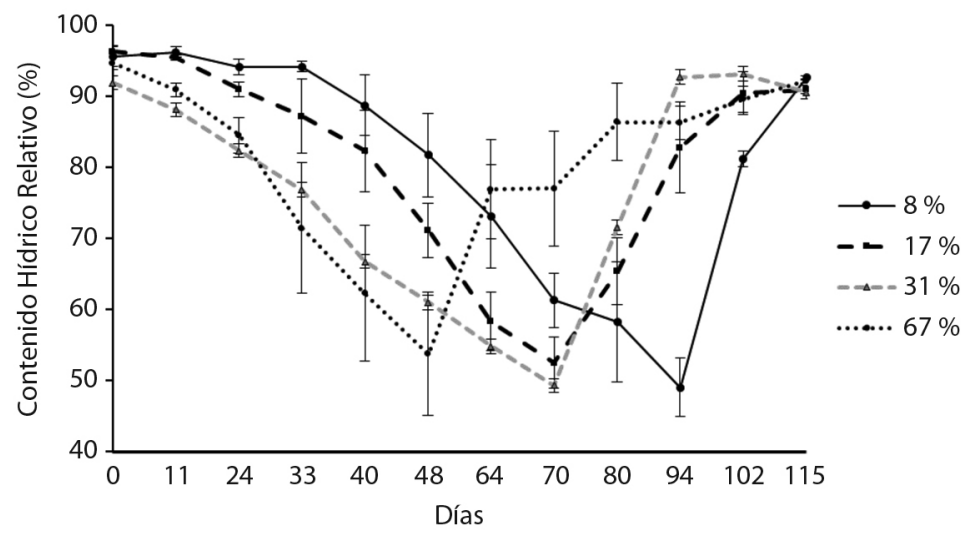

Fig. 1. Cambios en el contenido hídrico relativo $(\mathrm{CHR})$ promedio $(\mathrm{N}=5)$ en los tratamientos de radiación durante el ensayo. El CHR disminuyó hasta alcanzar punto crítico de deshidratación del tejido foliar (CHR $\leq 50 \% \pm 3$ ); luego en cada tratamiento se reanudó el riego hasta los 115 días del ensayo.

Fig. 1. Changes in average relative water content $(\mathrm{CHR})(\mathrm{N}=5)$ in radiation treatments during the trial. The CHR decreases until reaching the dehydration critical point of the leaf tissue (CHR $\leq 50 \% \pm 3)$; then in each treatment irrigation is resumed until 115 days of the test. 
TABLA 1

Efecto del tratamiento de radiación (IR \%) sobre la acumulación nocturna de ácidos ( $\mu$ equiv $\mathrm{H}^{+} \mathrm{g}^{-1}$ ), la eficiencia cuántica máxima ( $\mathrm{Fv} / \mathrm{Fm})$ y la asimilación de $\mathrm{CO}_{2}\left(\mathrm{mmol} \mathrm{m}^{-2}\right)$ diurna, nocturna y durante $24 \mathrm{~h}$, en las diferentes fases de deshidratación y rehidratación

TABLE 1

Effect of the relative illumination treatment (IR \%) on the nocturnal accumulation of acids ( $\mu$ equiv $\mathrm{H}^{+} \mathrm{g}^{-1}$ ), the maximum quantum efficiency $(\mathrm{Fv} / \mathrm{Fm})$ and the assimilation of $\mathrm{CO}_{2}\left(\mathrm{mmol} \mathrm{m}^{-2}\right)$ at daytime, nighttime and along $24 \mathrm{hs,} \mathrm{in} \mathrm{the} \mathrm{different} \mathrm{stages} \mathrm{of} \mathrm{dehydration} \mathrm{and} \mathrm{rehydration}$

\begin{tabular}{lccccccc}
\multicolumn{1}{c}{ Fase } & IR \% & CHR \% & $\begin{array}{c}\text { Acumul. } \\
\text { noct. ácidos } \\
\text { eequiv. H+ }\end{array}$ & $\begin{array}{c}\mathrm{FV} / \mathrm{Fm} \\
(7: 00 \mathrm{am})\end{array}$ & $\begin{array}{c}\text { Asimil. } \\
\text { diurna } \\
\mathrm{mmol} \mathrm{m} \mathrm{m}^{-2}\end{array}$ & $\begin{array}{c}\text { Asimil. nocturna } \\
\mathrm{mmol} \mathrm{m}^{-2}\end{array}$ & $\begin{array}{c}\text { Asimil. } 24 \mathbf{h} \\
\mathrm{mmol}^{-2}\end{array}$ \\
Hidrat. & 8 & $95.5 \pm 2 \mathbf{c}$ & $102 \pm 2.8 \mathbf{c}$ & $0.81 \pm 0.01 \mathbf{b}$ & $9.36 \pm 3.84 \mathbf{b}$ & $29.20 \pm 3.90 \mathbf{a b}$ & $38.56 \pm 5.55 \mathbf{a b}$ \\
óptima & 17 & $96.2 \pm 2 \mathbf{c}$ & $115 \pm 5.0 \mathbf{c}$ & $0.79 \pm 0.01 \mathbf{b}$ & $8.31 \pm 1.11 \mathbf{a b}$ & $41.21 \pm 5.32 \mathbf{b}$ & $49.52 \pm 6.29 \mathbf{b}$ \\
& 31 & $93.4 \pm 3 \mathbf{c}$ & $102 \pm 3.3 \mathbf{c}$ & $0.80 \pm 0.01 \mathbf{b}$ & $5.37 \pm 0.72 \mathbf{a b}$ & $36.83 \pm 7.23 \mathbf{b}$ & $42.21 \pm 7.01 \mathbf{b}$ \\
& 67 & $94.4 \pm 2 \mathbf{c}$ & $106 \pm 8.0 \mathbf{c}$ & $0.74 \pm 0.02 \mathbf{a}$ & $2.42 \pm 1.54 \mathbf{a}$ & $19.99 \pm 4.02 \mathbf{a}$ & $22.41 \pm 5.50 \mathbf{a}$ \\
Deshidrat. & 8 & $79.7 \pm 3 \mathbf{a}$ & $61 \pm 5.6 \mathbf{b}$ & $0.71 \pm 0.02 \mathbf{b}$ & $-7.82 \pm 0.41 \mathbf{b}$ & $12.34 \pm 9.16 \mathbf{b}$ & $4.52 \pm 5.15 \mathbf{b}$ \\
48 días & 17 & $71.1 \pm 3 \mathbf{a}$ & $47 \pm 9.4 \mathbf{a b}$ & $0.74 \pm 0.01 \mathbf{b}$ & $-1.21 \pm 0.46 \mathbf{c}$ & $4.01 \pm 5.64 \mathbf{a b}$ & $2.81 \pm 5.64 \mathbf{b}$ \\
& 31 & $60.1 \pm 3 \mathbf{b}$ & $31 \pm 3.3 \mathbf{a}$ & $0.72 \pm 0.02 \mathbf{b}$ & $-3.03 \pm 0.97 \mathbf{c}$ & $-3.54 \pm 1.74 \mathbf{a b}$ & $-6.57 \pm 1.74 \mathbf{a b}$ \\
& 67 & $53.3 \pm 3 \mathbf{a}$ & $32 \pm 3.8 \mathbf{a}$ & $0.62 \pm 0.04 \mathbf{a}$ & $-10.11 \pm 0.68 \mathbf{a}$ & $-14.14 \pm 7.58 \mathbf{a}$ & $-24.25 \pm 7.58 \mathbf{a}$ \\
Deshidrat. & 8 & $49.0 \pm 3 \mathbf{a}$ & $41 \pm 5.3 \mathbf{a}$ & $0.77 \pm 0.02 \mathbf{b}$ & $-7.93 \pm 1.51 \mathbf{a}$ & $-7.88 \pm 1.24 \mathbf{a}$ & $-15.81 \pm 2.61 \mathbf{a b}$ \\
crítica & 17 & $52.5 \pm 3 \mathbf{a}$ & $31 \pm 2.8 \mathbf{a}$ & $0.77 \pm 0.01 \mathbf{b}$ & $-2.39 \pm 0.53 \mathbf{b}$ & $-7.46 \pm 0,30 \mathbf{a}$ & $-9.85 \pm 299 \mathbf{b}$ \\
& 31 & $49.3 \pm 3 \mathbf{a}$ & $31 \pm 2.7 \mathbf{a}$ & $0.69 \pm 0.01 \mathbf{a}$ & $-3.11 \pm 0.62 \mathbf{b}$ & $-10.29 \pm 2.07 \mathbf{a}$ & $-13.4 \pm 2.51 \mathbf{a b}$ \\
& 67 & $53.3 \pm 3 \mathbf{a}$ & $32 \pm 3.8 \mathbf{a}$ & $0.62 \pm 0.04 \mathbf{a}$ & $-10.11 \pm 0.68 \mathbf{a}$ & $-14.14 \pm 7.58 \mathbf{a}$ & $-24.25 \pm 7.58 \mathbf{a}$ \\
Rehidrat. & 8 & $93.5 \pm 2 \mathbf{b}$ & $54 \pm 4.9 \mathbf{a}$ & $0.81 \pm 0.01 \mathbf{b}$ & $-9.58 \pm 2.73 \mathbf{a}$ & $26.68 \pm 8.58 \mathbf{a b}$ & $17.1 \pm 6.22 \mathbf{a b}$ \\
22 días & 17 & $90.1 \pm 2 \mathbf{b}$ & $106 \pm 4.3 \mathbf{c}$ & $0.77 \pm 0.01 \mathbf{b}$ & $-0.65 \pm 0.80 \mathbf{b c}$ & $35.55 \pm 7.14 \mathbf{b}$ & $34.9 \pm 7.91 \mathbf{b}$ \\
& 31 & $92.6 \pm 2 \mathbf{b}$ & $76 \pm 2.5 \mathbf{b}$ & $0.79 \pm 0.01 \mathbf{b}$ & $4.26 \pm 2.08 \mathbf{c}$ & $30.52 \pm 7.90 \mathbf{b}$ & $34.78 \pm 6.71 \mathbf{b}$ \\
& 67 & $76.8 \pm 2 \mathbf{a}$ & $51 \pm 9.9 \mathbf{a}$ & $0.63 \pm 0.02 \mathbf{a}$ & $-1.46 \pm 0.721 \mathbf{b}$ & $7.42 \pm 4.70 \mathbf{a}$ & $5.96 \pm 4.05 \mathbf{a}$ \\
\hline
\end{tabular}

Promedios \pm Error estándar, para $\mathrm{N}=$ cuatro plantas en las variables CHR \%; Fv / Fm; Asimil (diurna, nocturna, 24 h), y $\mathrm{N}$ = cinco plantas para la variable Acumul. noct. Ácidos. Los promedios con la misma letra en una columna de cada fase del experimento no son significativamente diferentes entre sí $(\mathrm{P}<0.05)$ usando una prueba Rangos Múltiples de Duncan. IR \%: tratamiento de radiación; CHR \%: Contenido Hídrico Relativo; Fv / Fm: Eficiencia cuántica del fotosistema II: Acumul: acumulación; noct: nocturna (18:00 h- 06:00 h); Asimil: asimilación.

Una vez alcanzado el punto de deshidratación crítica en todos los tratamientos $(\mathrm{CHR} \leq 50 \%)$, continuó la tendencia en la asimilación diurna de $\mathrm{CO}_{2}$ con valores significativamente superiores $(\mathrm{P} \leq 0.05)$ de los tratamientos de iluminación intermedia comparados con los tratamientos extremos, que no mostraron diferencias entre ellos. En esta fase no hubo diferencias significativas en los valores de asimilación nocturna de $\mathrm{CO}_{2}$ entre los tratamientos. En consecuencia, el balance de $\mathrm{CO}_{2}$ durante el período de 24 horas fue más favorable para el tratamiento del $17 \%$, siendo significativamente diferente del tratamiento del $67 \%$ (Fig. 2C).
Después del período de rehidratación (22 \pm 2 días) para cada uno de los tratamientos, los menores valores de asimilación diurna de $\mathrm{CO}_{2}$ se presentaron en el tratamiento de menor radiación, con diferencias significativas con respecto a los demás tratamientos: La mayor recuperación, con valores más altos de asimilación nocturna de $\mathrm{CO}_{2}$, la alcanzaron los tratamientos intermedios y esta misma tendencia se expresó en el balance de fotosíntesis durante 24 horas. En todos los tratamientos ocurrió un desplazamiento temporal de la asimilación máxima de $\mathrm{CO}_{2}$ a lo largo de las diferentes fases del ensayo. Esto se hizo más notorio entre la fase de hidratación óptima y la fase 

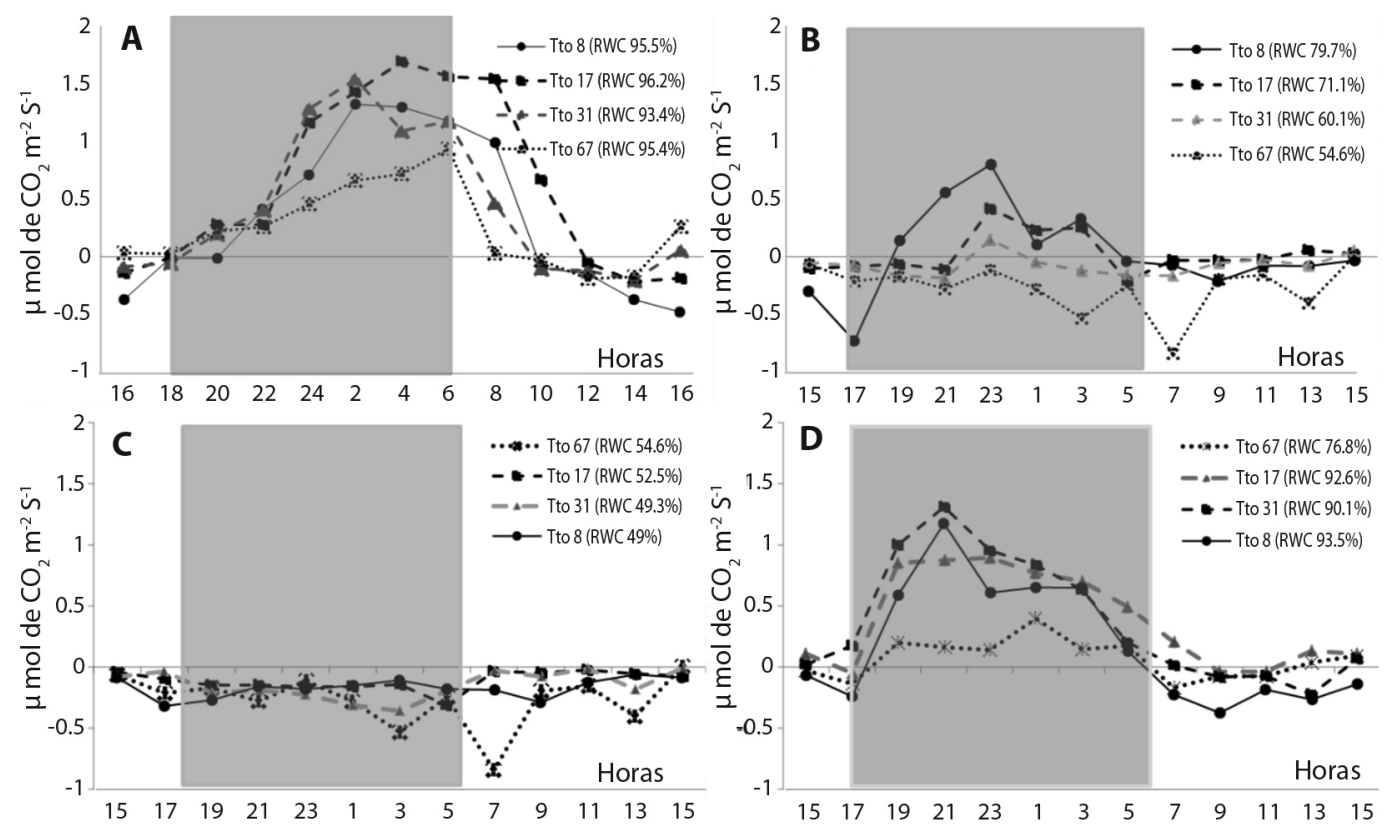

Fig. 2. Patrones diarios de asimilación de $\mathrm{CO}_{2}\left(\mu \mathrm{mol} \mathrm{m} \mathrm{m}^{-2} \mathrm{~s}^{-1}\right)$ de plantas de vainilla bajo distintas condiciones de radiación durante las fases de deshidratación y rehidratación: A. Condición inicial: plantas bien hidratadas; B. Después de 48 días de sequía prolongada, C. Cuando alcanzaron el punto crítico de deshidratación (CHR $\leq 50 \% \pm 3)$ en todos los tratamientos de iluminación; D. Después de $22 \pm 2$ días de rehidratación en cada uno de los tratamientos. Las franjas grises representan las horas de oscuridad. Los valores en paréntesis en la leyenda indican el contenido hídrico relativo promedio $(\mathrm{N}=5)$.

Fig. 2. Daily $\mathrm{CO}_{2}$ assimilation patterns $\left(\mu \mathrm{mol} \mathrm{m} \mathrm{m}^{-2} \mathrm{~s}^{-1}\right)$ of vanilla plants under different radiation conditions during the dehydration and rehydration phases: A. Initial condition: well-hydrated plants; B. After 48 days of prolonged drought, C. When they reached the critical point of dehydration (CHR $\leq 50 \% \pm 3$ ) in all lighting treatments; D. After $22 \pm 2$ days of rehydration in each of the lighting treatments. The gray stripes represent the hours of darkness. Values in parentheses in the legend indicate the average relative water content $(\mathrm{N}=5)$.

de rehidratación, ya que la máxima asimilación ocurrió antes del amanecer (Fig. 2A) y antes de la media noche (Fig. 2D), respectivamente.

\section{Cambios en la acumulación nocturna} de ácidos durante la deshidratación y rehidratación de las plantas: $\mathrm{Al}$ inicio del ensayo, durante la fase de hidratación óptima (CHR $\geq 90 \%$ ) no hubo diferencias en la acumulación nocturna de ácidos orgánicos (Tabla 1). Después de 48 días de sequía, se evidenciaron diferencias entre los tratamientos: el tratamiento con menor radiación fue diferente a los tratamientos del 31 y $67 \%(\mathrm{P} \leq 0.05)$, los cuales no fueron diferentes entre sí, ni con el tratamiento del $17 \%$; pero cuando se alcanzó la deshidratación crítica, esta sequía extrema redujo la capacidad de acumulación nocturna de ácidos orgánicos con respecto a su condición inicial en todos los tratamientos (reducción mayor al $60 \%$ ), aunque los valores promedios $(\mathrm{N}=5)$ no presentaron diferencias significativas entre ellos $(\mathrm{P}>0.05)$ (Tabla 1).

Después de la rehidratación de las plantas durante 22 días en cada tratamiento, la acumulación nocturna de ácidos se recuperó, con diferencias entre los tratamientos $\mathrm{y}$, fue significativamente menor $(\mathrm{P} \leq 0.05)$ en los tratamientos de menor y mayor radiación, con valores de recuperación de $53.6 \%$ y $47.2 \%$, respectivamente, los cuales no difirieron entre sí $(\mathrm{P}>0.05)$; en contraste, los tratamientos intermedios, se recuperaron por encima del $80 \%$, siendo significativamente superior 
$(\mathrm{P} \leq 0.05)$ el valor alcanzado por las plantas del tratamiento del $17 \%$ de IR (Tabla 1).

Cambios en la fluorescencia de la clorofila $(\mathrm{Fv} / \mathrm{Fm})$ durante la deshidratación y rehidratación de las plantas: Durante la fase óptima de hidratación en la primera fase del ensayo, Fv/Fm del tratamiento con mayor radiación fue significativamente inferior a los otros tratamientos, y este mismo patrón continuó después de 48 días de sequía (Tabla 1). Cuando las plantas de todos los tratamientos alcanzaron el punto crítico de deshidratación, el valor del tratamiento de $31 \%$ cayó por debajo de 0.7 ; este, junto con el tratamiento del $67 \%$ fueron significativamente inferiores a los tratamientos más sombreados. Después de la rehidratación de las plantas, los valores aumentaron en todos los tratamientos, aunque el tratamiento más iluminado siguió siendo el más afectado, con diferencias significativas con respecto a los demás tratamientos $(\mathrm{P} \leq$ $0.05)$, mientras que el tratamiento del $31 \%$ logró recuperarse (Tabla 1).

\section{DISCUSIÓN}

La vainilla es un cultivo recomendado para muchas zonas bajas de los trópicos, donde se requieren actividades sostenibles que se puedan incorporar a los sistemas agroforestales o al manejo de bosques secundarios, con posibilidades de generar ingresos para las comunidades locales (Brownell, 2011). Al ser una planta CAM, tiene mayores oportunidades de sobrellevar los efectos adversos de la sequía, al ser más eficiente en el uso del agua (Töpfer, Braam, Shameer, Ratcliffe, \& Sweetlove, 2020). Sin embargo, al igual que otros cultivos, esta opción puede verse amenazada por la disminución y la mayor estacionalidad en la precipitación bajo escenarios futuros de cambio climático (Gitz, Meybeck, Lipper, Young, \& Braatz, 2016).

En este estudio, las plantas de vainilla mostraron siempre fotosíntesis y funcionamiento del CAM en hojas maduras lo cual confirma su condición de especie CAM obligada o constitutiva (Grifths, 1989), aunque modificaron las proporciones de asimilación nocturnadiurna de $\mathrm{CO}_{2}$, reguladas por las condiciones ambientales prevalentes. Además, bajo condiciones óptimas de hidratación, en todos los tratamientos de radiación, las plantas de vainilla exhibieron una fotosíntesis y funcionamiento del CAM tipo 'fuerte', con mayor proporción de la asimilación de $\mathrm{CO}_{2}$ durante la noche; pero también presentaron asimilación diurna (Silvera et al., 2005) y las típicas cuatro fases del modelo de fotosíntesis y funcionamiento del CAM propuestas por Osmond (1978).

El resultado de que los valores de asimilación de $\mathrm{CO}_{2}$ durante 24 horas en las plantas de vainilla bien hidratadas fueran menores en el caso del tratamiento de mayor radiación, equivalente a $1340 \mu \mathrm{mol} \mathrm{m} \mathrm{seg}^{-1}$, coincide con lo reportado anteriormente, pues en estas condiciones ocurren procesos foto-inhibitorios que desencadenan en daños oxidativos permanentes en los fotosistemas (Puthur, 2005; Díez et al., 2017). La alta radiación puede inactivar los centros de reacción, inhibir el transporte de electrones a través de los fotosistemas, afectar las actividades de las enzimas del ciclo de carbono y en consecuencia las tasas de asimilación de $\mathrm{CO}_{2}$ (Netto, Campostrini, de Oliveira, \& Bressan-Smith, 2005). Esto explica por qué en las plantas que crecieron en esta radiación se redujo la asimilación de $\mathrm{CO}_{2}$ y se incrementó la respiración en las horas de luz. La tendencia a fotoinhibición de la vainilla en el tratamiento de alta radiación también se evidenció con los menores valores de Fv/Fm. En plantas bien hidratadas, la similitud en la acumulación nocturna de ácidos entre ambientes lumínicos, probablemente se debe a que la apertura estomática durante la fase I (carboxilación) no se vio afectada por el ambiente lumínico en el que crecieron las plantas. Otras orquídeas epifitas durante la temporada de lluvias, tampoco presentaron diferencias entre sí en esta variable, independientemente del ambiente lumínico en el que crecieron (De la Rosa, Andrade, Zotz, \& Reyes-García, 2014). De manera similar, en pitahaya (Hylocereus undatus), una planta CAM, trepadora y tolerante a la sombra, 
la acumulación nocturna de ácidos fue más sensible a los cambios de humedad que a los cambios de radiación (Andrade et al., 2006).

Cuando se interrumpió el riego, en los tratamientos con menor radiación se mantuvo un alto CHR durante las primeras semanas de sequía, lo cual se debe probablemente a las menores temperaturas asociadas, tanto foliares como del aire, menores tasas de transpiración y bajos valores de déficit de presión de vapor (Holmgren, 2000; Cavatte et al., 2012). Esto se hizo evidente en el tiempo que tardaron las plantas de cada tratamiento para alcanzar el punto crítico de deshidratación (CHR $\leq 50 \% \pm$ 3 ), con una diferencia considerable de 46 días, entre el tratamiento de menor y el de mayor radiación; pero aún las plantas que crecieron en el ambiente de mayor radiación tardaron cerca de mes y medio para alcanzarlo. Esta alta resistencia a la sequía probablemente se debe además a que las hojas de vainilla son suculentas, con una hipodermis que funciona como depósito de agua y que puede ocupar hasta el $90 \%$ de su volumen, como ocurre en otras especies de la familia Orchidaceae (Zotz \& Hietz, 2001). Además, las hojas están recubiertas por una cutícula impermeable que tiene idioblastos (estructuras engrosadas que acumulan agua y evitan el colapso de los tejidos ante el déficit hídrico) (Sandoval-Zapotitla \& Terrazas, 2001). Durante el proceso de deshidratación, el efecto combinado de la sequía y la alta radiación indujo menores valores de $\mathrm{Fv} / \mathrm{Fm}$ en los tratamientos de mayor radiación. Por ejemplo, después de 48 días de sequía, fue visible la afectación sobre el tratamiento de mayor radiación (valores de Fv/Fm de 0.65-0.62), lo cual indica la ocurrencia de fotoinhibición moderada y por tanto reducción de la captura de $\mathrm{CO}_{2}$ debido a los daños de los centros de reacción del PII (Adams, Zarter, Mueh, \& DemmigAdams, 2006). No obstante, al llegar al punto crítico de deshidratación, el tratamiento del 31 $\%$ también tuvo valores por debajo de 0.7 , lo cual una vez más sugiere la mayor vulnerabilidad a la fotoinhibición en las plantas sometidas a radiación media, bajo condiciones de sequía prolongada. Esto indica que la reducción en la captura de luz por los fotosistemas, no es una respuesta inmediata al estrés hídrico, salvo en condiciones de sequía severa, bajo la cual se producen procesos de fotoinhibición que pueden conducir a la destrucción oxidativa de los fotosistemas (Flexas, Gallé, Galmés, RibasCarbo, \& Medrano, 2012).

Después de mes y medio de sequía, en todos los tratamientos, el balance de la asimilación de $\mathrm{CO}_{2}$ durante 24 horas disminuyó más del $70 \%$ del valor inicial registrado bajo hidratación óptima. Estos efectos se incrementaron cuando se alcanzó el punto crítico de deshidratación, pues la capacidad de asimilación cayó drásticamente (disminuyó más del $110 \%$ ), con mayor reducción en el tratamiento de mayor radiación. Como ya se mencionó, la vainilla carece de mecanismos eficientes para tolerar y disipar el exceso de radiación (Graham \& Andrade, 2004), lo que explica su mayor afectación bajo tales condiciones. En el otro extremo del rango de radiación, después de 48 días de sequía, el tratamiento con menor radiación tuvo la mayor tasa de asimilación en el período de 24 horas debido principalmente a valores más altos de asimilación nocturna. Parecería que esto no concuerda con nuestra predicción de mayor afectación en los tratamientos extremos de radiación ( $8 \%$ y $67 \%$ ). No obstante, bajo condiciones de deshidratación crítica, desapareció la ventaja del tratamiento con menor radiación, y su balance de asimilación fue inferior al de los tratamientos de 17 y $31 \%$, confirmando nuestra predicción.

En cuanto a las fases de fotosíntesis y funcionamiento del CAM propuestas por Osmond (1978), en las plantas de vainilla luego de un período corto de sequía (cuando la disminución del CHR fue leve o moderada), el patrón de las fases metabólicas se ajustó de tal manera que la fase I (asimilación nocturna de $\mathrm{CO}_{2}$ y la formación de ácidos orgánicos) se vio seriamente reducida, y desaparecieron las fases II (aumento en la conductancia estomática y en la asimilación de $\mathrm{CO}_{2}$, al amanecer) y IV (donde el $\mathrm{CO}_{2}$ endógeno y exógeno es fijado por la Rubisco y por la PEPC, en el día). Es probable que tal comportamiento obedezca a un mecanismo 
fisiológico de resistencia relacionado con el cierre de estomas, los cuales son altamente sensibles a la de pérdida de agua (Taiz \& Zeiger, 2006). Cuando el mesófilo comienza a sufrir deshidratación, el contenido de ácido abscísico (ABA) en la hoja se incrementa debido a la des-compartimentalización y redistribución desde los cloroplastos de las células del mesófilo hasta las células de guarda de las estomas (Zhang \& Outlaw, 2001). Este proceso influye en la apertura estomática y por ende en la proporción de $\mathrm{CO}_{2}$ fijado bajo condiciones de estrés hídrico leve o moderado. Los resultados de asimilación muestran que bajo condiciones de estrés hídrico severo $(\mathrm{CHR} \leq 50 \% \pm 3)$, las estomas permanecieron cerrados todo el día y toda la noche, probablemente como mecanismo para evitar la pérdida de agua, lo que a su vez evitó la absorción de $\mathrm{CO}_{2}$. No obstante, ocurrió acumulación nocturna de ácidos, aunque en bajas cantidades. Este comportamiento ha sido reportado por Lüttge (2006) como característico de la variante denominada CAM idling, en la cual las estomas se cierran completamente cuando la sequía es severa y el $\mathrm{CO}_{2}$ respiratorio es re-fijado en la noche y reciclado a carbohidratos durante el día. La variante CAM-idling se considera como una forma característica de CAM muy fuerte, presente en especie de hojas suculentas (Lüttge, 2004) como la vainilla, que tienen alta capacidad de almacenamiento de agua en los tejidos. La inducción de CAMidling es considerada como una respuesta al estrés, sin absorción neta de $\mathrm{CO}_{2}$ (Dodd, Borland, Haslam, Griffiths, \& Maxwell, 2002).

Después de volver a regar las plantas de cada tratamiento durante 22 días, en todos los casos se recuperó más del $90 \%$ del CHR, excepto en el tratamiento de mayor radiación, y lo mismo ocurrió con la eficiencia cuántica, lo cual indica un daño crónico en las plantas. Se ha reportado que en vainilla la exposición a altas cantidades de radiación provoca clorosis en gran parte de las hojas, como evidencia de una lesión irreversible (Puthur, 2005), y es posible que, bajo estrés hídrico severo acompañado de alta radiación, se produzcan daños duraderos que afectan los centros de reacción del PSII y causen foto destrucción de los pigmentos fotosintéticos (Graham \& Andrade, 2004; Flexas et al., 2012;). En todos los tratamientos se recuperó parcialmente la capacidad de fijación de $\mathrm{CO}_{2}$ durante 24 horas y la acumulación de ácidos orgánicos, aunque leve en los tratamientos extremos y, de mayor magnitud, en los intermedios. Esto se debe probablemente a que, una vez desaparece el estrés hídrico y hay buen suministro de agua desde las raíces, se recupera la turgencia de los tejidos, las estomas vuelven a abrirse y ocurren cambios metabólicos que posibilitan una rápida recuperación de la fotosíntesis y del funcionamiento del CAM (Flexas et al., 2012) y, en consecuencia, se incrementa la transformación en ácidos orgánicos durante la noche (Dodd et al., 2002). Estos resultados apoyan nuestra predicción de que la radiación fotosintéticamente activa muy baja o muy alta en los tratamientos extremos de radiación son limitantes para los procesos fisiológicos de la vainilla.

Declaración de ética: los autores declaran que todos están de acuerdo con esta publicación y que han hecho aportes que justifican su autoría; que no hay conflicto de interés de ningún tipo; y que han cumplido con todos los requisitos y procedimientos éticos y legales pertinentes. Todas las fuentes de financiamiento se detallan plena y claramente en la sección de agradecimientos. El respectivo documento legal firmado se encuentra en los archivos de la revista.

\section{RESUMEN}

Introducción: La vainilla (Vanilla planifolia Andrews) es una planta CAM de gran importancia económica en el mercado mundial de saborizantes y aromatizantes. Por ser una planta hemiepífita que inicialmente crece en el sotobosque sombreado y posteriormente asciende hasta el dosel, se aclimata a diferentes condiciones de radiación y humedad. La posibilidad de extender su cultivo a zonas secas donde sea menor la incidencia de enfermedades, dependerá de su respuesta a períodos prolongados de sequía. Objetivo: Evaluar los efectos de la interacción entre la radiación y la humedad sobre los parámetros de la fotosíntesis y funcionamiento del CAM de plantas de vainilla en sus fases iniciales de desarrollo vegetativo, y 
determinar si las condiciones de radiación en las que crece la planta pueden ayudar a moderar los efectos negativos de la sequía. Métodos: Se evaluaron plantas de vainilla que crecieron con riego durante 18 meses en casetas con iluminación relativa (IR) de $8,17,31$ y $67 \%$; posteriormente se suspendió el riego y se fueron deshidratando durante 94 días hasta alcanzar un contenido de humedad crítico (contenido hídrico relativo, $\mathrm{CHR} \leq 50 \%$ ), y finalmente se reinició el riego durante 22 días; se evaluó el efecto de estos procesos sobre la asimilación de $\mathrm{CO}_{2}$, la acidez titulable y la eficiencia cuántica del Fotosistema II (Fv/ Fm). Resultados: bajo condiciones intermedias de IR (17 $\%$ y $31 \%$ ), la asimilación de $\mathrm{CO}_{2}$ y la acumulación nocturna de ácidos orgánicos presentaron la menor afectación por el déficit hídrico, así como la mejor recuperación luego de la rehidratación. El CHR se vio más afectado por la disponibilidad de agua en el tejido vegetal, mientras que Fv/ Fm lo fue por el ambiente de radiación. Los efectos combinados de estrés hídrico y alta radiación ocasionaron daños irreversibles en la fotosíntesis para el tratamiento de IR de $67 \%$. Conclusiones: En las plantas de vainilla, el impacto negativo de la sequía sobre los parámetros de la fotosíntesis fue mayor en alta radiación; sin embargo, en condiciones de baja radiación también aumentó la susceptibilidad de las plantas a la sequía, en comparación con los ambientes de radiación intermedia, en los cuales la densidad de flujo de fotones media fue de $340 \mu \mathrm{mol} \mathrm{m} \mathrm{seg}^{-1}$ (17\% de IR) y $620 \mu \mathrm{mol} \mathrm{m} \mathrm{m}^{-2} \mathrm{seg}^{-1}(31 \%$ de IR). Estos resultados sugieren el potencial de cultivar vainilla en zonas con sequía estacional bajo sistemas productivos de baja tecnificación, manteniendo estas condiciones de radiación.

Palabras clave: acidez titulable; CAM; contenido hídrico relativo; déficit hídrico; fluorescencia de la clorofila; Orchidaceae.

\section{REFERENCIAS}

Adams III, W.W., Zarter, C.R., Mueh, K.E., \& DemmigAdams, B. (2006). Energy dissipation and photoinhibition: a continuum of photoprotection. En B. Demmig-Adams, W.W. Adams III, \& A.K. Mattoo (Eds.), Photoprotection, photoinhibition, gene regulation, and environment (pp. 49-64). Dordrecht, Netherlands: Springer.

Andrade, J.L., Rengifo, E., Ricalde, M.F., Simá, J.L., Cervera, J.C., \& Soto, G.V. (2006). Microambientes de luz, crecimiento y fotosíntesis de la pitahaya (Hylocereus undatus) en un agrosistema de Yucatán, México. Agrociencia, 40(6), 687-697.

Borland, A.M., Maxwell, K., \& Griffiths, H. (2000) Ecophysiology of the CAM pathway. In R.C. Leegood, T.D. Sharkey, \& S. von Caemmerer (Eds.), Advances in Photosynthesis. Photosynthesis: Physiology and Metabolism (pp. 583-605). Dordrecht, Netherlands: Kluwer Academic Publishers.
Bory, S., Grisoni, M., Duval, M.F., \& Besse, P. (2008). Biodiversity and preservation of vanilla: present state of knowledge. Genetic Resources and Crop Evolution, 55(4), 551-571.

Brownell Jr., R.J. (2011). Fair Trade-the future of vanilla. En D. Havkin-Frenkel \& F.C. Belanger (Eds.), Handbook of vanilla science and technology (pp. 107-116). West Sussex, UK: Blackwell Publishing Ltd.

Buss, A. (2020). Como o gradiente altitudinal de luz no sub-bosque afeta as características estruturais e fisiológicas de hemiepífitas: o caso de Vanilla bahiana Hoehne (Orchidaceae) (Mestria em Biologia Vegetal). Universidade Federal de Uberlândia, Uberlândia, MG, Brasil.

Cavatte, P.C., Oliveira, Á.A., Morais, L.E., Martins, S.C., Sanglard, L.M., \& Da Matta, F.M. (2012). Could shading reduce the negative impacts of drought on coffee? A morphophysiological analysis. Physiologia Plantarum, 144(2), 111-122.

Ceusters, J., Borland, A.M., Londers, E., Verdoodt, V., Godts, C., \& De Proft, M.P. (2009). Differential usage of storage carbohydrates in the CAM bromeliad Aechmea 'Maya' during acclimation to drought and recovery from dehydration. Physiologia Plantarum, 135(2), 174-184.

de la Rosa-Manzano, E., Andrade, J.L., Zotz, G., \& Reyes-García, C. (2017). Physiological plasticity of epiphytic orchids from two contrasting tropical dry forests. Acta Oecologica, 85, 25-32.

Díez, M.C., Moreno, F., \& Gantiva, E. (2017). Effects of light intensity on the morphology and CAM photosynthesis of Vanilla planifolia Andrews. Revista Facultad Nacional de Agronomía Medellín, 70(1), 8023-8033.

Dodd, A.N., Borland, A.M., Haslam, R.P., Griffiths, H., \& Maxwell, K. (2002). Crassulacean acid metabolism: plastic, fantastic. Journal of Experimental Botany, 53(369), 569-580.

Flexas, J., Gallé, A., Galmés, J., Ribas-Carbo, M., \& Medrano, H. (2012). The response of photosynthesis to soil water stress. In R. Aroca (Eds.), Plant Responses to Drought Stress (pp. 129-144). Berlin, Heidelberg: Springer.

Fouché, J.G., \& Jouve, L. (1999). Vanilla planifolia: history, botany and culture in Reunion Island. Agronomie, 19(8), 689-703.

Fu, C.F., \& Hew, C.S. (1982). Crassulacean acid metabolism in orchids under water stress. Botanical Gazette, 143(3), 294-297.

Gitz, V., Meybeck, A., Lipper, L., Young, C.D., \& Braatz, S. (2016). Climate change and food security: risks and responses. Rome, Italy: Food and Agriculture Organization of the United Nations (FAO). 
Graham, E.A., \& Andrade, J.L. (2004). Drought tolerance associated with vertical stratification of two co-occurring epiphytic bromeliads in a tropical dry forest. American Journal of Botany, 91(5), 699-706.

Griffiths, H. (1989). Crassulacean acid metabolism: a re-appraisal of physiological plasticity in form and function. In J.A. Callow (Ed.), Advances in Botanical Research (pp. 43-92). London, UK: Academic Press.

Haslam, R., Borland, A., Maxwell, K., \& Griffiths, H. (2003). Physiological responses of the CAM epiphyte Tillandsia usneoides L. (Bromeliaceae) to variations in light and water supply. Journal of Plant Physiology, 160(6), 627-634.

Hernández, J. (2011). Mexican Vanilla Production. In D. Havkin-Frenkel, \& F. Belanger (Eds.), Handbook of Vanilla Science and Technology (pp. 1-25). West Sussex, UK: Wiley-Blackwell.

Holmgren, M. (2000). Combined effects of shade and drought on tulip poplar seedlings: trade-off in tolerance or facilitation? Oikos, 90(1), 67-78.

Kadir, N.A., Naher, L., \& Sidek, N. (2019). Economical important phytopathogenic diseases in Vanilla planifolia. Journal of Tropical Resources and Sustainable Science, 7, 77-82.

Lüttge, U. (2004). Ecophysiology of crassulacean acid metabolism(CAM). Annals of Botany, 93(6), 629-652.

Lüttge, U. (2006). Photosynthetic flexibility and ecophysiological plasticity: questions and lessons from Clusia, the only CAM tree, in the neotropics. New Phytologist, 171(1), 7-25.

Markesteijn, L., \& Poorter, L. (2009). Seedling root morphology and biomass allocation of 62 tropical tree species in relation to drought and shade tolerance. Journal of Ecology, 97(2), 311-325.

Munns, R. (2010). Water relations. Recuperado de http://prometheuswiki.org/tiki-index. php?page $=$ Water + relations

Netto, A.T., Campostrini, E., de Oliveira, J.G., \& Bressan-Smith, R.E. (2005). Photosynthetic pigments, nitrogen, chlorophyll a fluorescence and SPAD502 readings in coffee leaves. Scientia Horticulturae, 104(2), 199-209.

Niinemets, Ü., \& Valladares, F. (2006). Tolerance to shade, drought, and waterlogging of temperate Northern Hemisphere trees and shrubs. Ecological Monographs, 76(4), 521-547.

Osmond, C.B. (1978). Crassulacean Acid Metabolism: a curiosity in context. Annual Review of Plant Physiology, 29(1), 379-414.
Puthur, J. (2005). Influence of light intensity on growth and crop productivity of Vanilla planifolia Andr. General and Applied Plant Physiology, 31(3-4), 215-224.

Ramírez, C., Rapidel, B., \& Mattey, J. (1999). Principales factores agronómicos en el cultivo de la vainilla y su alivio en la zona de Quepos, Costa Rica. En Memorias del XI Congreso Nacional Agronómico, San José, Costa Rica.

Sack, L., \& Grubb, P.J. (2002). The combined impacts of deep shade and drought on the growth and biomass allocation of shade-tolerant woody seedlings. Oecologia, 131(2), 175-185.

Sandoval-Zapotitla, E., \& Terrazas, T. (2001). Leaf anatomy of 16 taxa of the Trichocentrum clade (Orchidaceae: Oncidiinae). Lindleyana, 16, 81-93.

Silvera, K., Santiago, L.S., \& Winter, K. (2005). Distribution of crassulacean acid metabolism in orchids of Panama: evidence of selection for weak and strong modes. Functional Plant Biology, 32, 397-407.

Silvera, K., \& Lasso, E. (2016). Ecophysiology and crassulacean acid metabolism of tropical epiphytes. In G. Goldstein, \& L.S. Santiago (Eds.), Tropical tree physiology: adaptations and responses in a changing environment (pp. 25-43). New York, USA: Springer.

Taiz, L., \& Zeiger, E. (2006). Plant Physiology. Fourth Edition. New York, USA: Sinauer Associates.

Tay, S., He, J., \& Yam, T.W. (2019). CAM plasticity in epiphytic tropical orchid species responding to environmental stress. Botanical Studies, 60(1), 7.

Töpfer, N., Braam, T., Shameer, S., Ratcliffe, R.G., \& Sweetlove, L.J. (2020). CAM emerges in a leaf metabolic model under water-saving constraints in different environments. bioRxiv

Winter, K. (2019). Ecophysiology of constitutive and facultative CAM photosynthesis. Journal of Experimental Botany, 70(22), 6495-6508.

Zhang, S.Q., \& Outlaw, W.H. (2001). Abscisic acid introduced into the transpiration stream accumulates in the guard cell apoplast and causes stomatal closure. Plant and Cell Environment, 24, 1045-1054.

Zotz, G. (2004). How prevalent is crassulacean acid metabolism among vascular epiphytes? Oecologia, 138(2), 184-192.

Zotz, G., \& Hietz, P. (2001). The physiological ecology of vascular epiphytes current knowledge, open questions. Journal of Experimental Botany, 52(364), 2067-2078. 